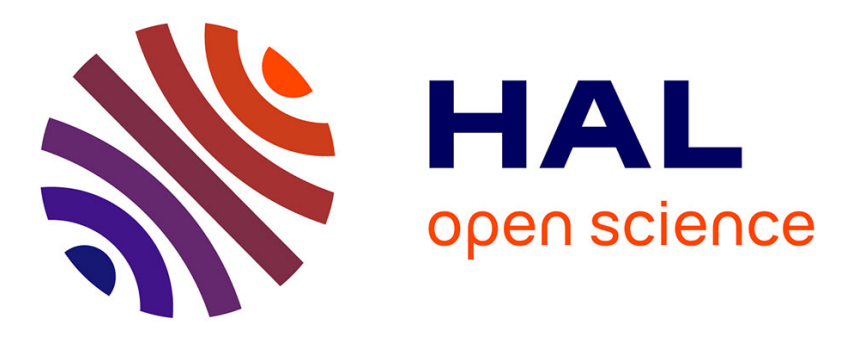

\title{
Energy balance in surface nanosecond dielectric barrier discharge. Plasma-assisted ignition of heavy hydrocarbons at high pressures
}

\author{
S.A. Shcherbanev, S.A. Stepanyan, Mohamed Boumehdi, Guillaume Vanhove, \\ Pascale Desgroux, Svetlana Starikovskaia
}

\section{To cite this version:}

S.A. Shcherbanev, S.A. Stepanyan, Mohamed Boumehdi, Guillaume Vanhove, Pascale Desgroux, et al.. Energy balance in surface nanosecond dielectric barrier discharge. Plasma-assisted ignition of heavy hydrocarbons at high pressures. 53rd AIAA Aerospace Sciences Meeting, 2015, Kissimmee, France. pp.6118 - 6125, 10.2514/6.2015-0668 . hal-01738387

\section{HAL Id: hal-01738387 \\ https://hal.science/hal-01738387}

Submitted on 14 Sep 2020

HAL is a multi-disciplinary open access archive for the deposit and dissemination of scientific research documents, whether they are published or not. The documents may come from teaching and research institutions in France or abroad, or from public or private research centers.
L'archive ouverte pluridisciplinaire HAL, est destinée au dépôt et à la diffusion de documents scientifiques de niveau recherche, publiés ou non, émanant des établissements d'enseignement et de recherche français ou étrangers, des laboratoires publics ou privés. 


\title{
Energy balance in surface nanosecond dielectric barrier discharge. Plasma-assisted ignition of heavy hydrocarbons at high pressures.
}

\author{
S. A. Shcherbanev, S. A. Stepanyan ${ }^{\dagger}$ \\ Laboratory of Plasma Physics (CNRS, Ecole Polytechnique, Sorbonne Universities, \\ University of Pierre and Marie Curie-Paris 6, University Paris-Sud), \\ Route de Saclay, 91128, Palaiseau Cedex, France \\ M. A. Boumehdi $\ddagger$ G. Vanhove ${ }^{\S}$ and P. Desgroux ฯ \\ PC2A Lille University of Science and Technology, 59655, Villeneuve d'Ascq, France \\ S. M. Starikovskaia ॥ \\ Laboratory of Plasma Physics (CNRS, Ecole Polytechnique, Sorbonne Universities, \\ University of Pierre and Marie Curie-Paris 6, University Paris-Sud), \\ Route de Saclay, 91128, Palaiseau Cedex, France
}

\begin{abstract}
The paper presents experimental study of nanosecond surface dielectric barrier discharge (nSDBD) in air and application of nSDBD for initiation of the two-stage ignition of n-heptane in mixture with air. The emission spectroscopy study of rotational and vibrational structure of $2^{+}$system of $\mathrm{N}_{2}$ molecules has been performed for a classical air flow control SDBD configuration. The energy deposition into the discharge has been measured. Strongly non-equilibrium distribution of rotational population is observed in the spectra at the leading and trailing edges of the high-voltage pulse. The time resolved spatial distribution of the rotational temperature along the dielectric surface has been measured. The second part of the paper concerns the ignition of stoichiometric mixture of $\mathrm{C}_{7} \mathbf{H}_{16} / \mathrm{O}_{2} / \mathrm{N}_{2}$ with nanosecond SDBD in Rapid Compression Machine (RCM). Different regimes of ignition initiated by discharge are discussed, including cool flame and fast ignition.
\end{abstract}

\section{Introduction}

Transient plasma of nanosecond discharges is widely used for studying of the kinetic effects connected to plasma assisted ignition (PAI) and plasma assisted combustion (PAC) in hydrocarbon-containing mixtures. Unlike combustion process, very sensitive to initial temperature of the mixture, the discharge development is determined, first of all, by a gas number density. This is dictated by the fact that the mean energy of electrons is a function of reduced electric field, $E / N$, were $E$ is the electric field and $N$ is a number density of neutral particles responsible for the loss of electron energy in collisions. So, gas number densities must be analyzed when discussing the discharge efficiency.

*PhD student, Laboratory for Plasma Physics, Ecole Polytechnique, Palaiseau, France

${ }^{\dagger}$ PhD student, Laboratory for Plasma Physics, Ecole Polytechnique, Palaiseau, France

${ }^{\ddagger}$ PhD Student, Laboratory of Physics of Physico-Chemical Processes in Combustion and in Atmosphere, Lille University, France

§Senior Researcher, Laboratory of Physics of Physico-Chemical Processes in Combustion and in Atmosphere, Lille University, France

I Leading Researcher, Laboratory of Physics of Physico-Chemical Processes in Combustion and in Atmosphere, Lille University, France

" Leading Researcher, Laboratory for Plasma Physics, Ecole Polytechnique, Palaiseau, France 
The efficiency of plasma-assisted combustion is determined (i) by spatial-temporal distribution of energy deposited in the discharge; (ii) by values of reduced electric fields and electron density during the stage of the main energy release, and so, by composition of active species in the discharge and near afterglow. The electric fields are limited by different factors, such as voltage applied to the system, geometry of the electrodes or by density of charged species already produced in plasma.

Nanosecond discharges initiated at ambient gas temperature by pulses 10-100 kV in amplitude, a few nanoseconds rise time and a few tens of nanoseconds in duration, produce uniform plasma in a wide range of pressures, from units of Torr to parts of atm. ${ }^{1}$ At short distances between the electrodes, the pressure region where the glow-like discharge is observed can be extended to a few atm. ${ }^{2}$ Electric fields in the front of the discharge can be as high as a few kTd during a very short time, less than a few nanoseconds. After this, the fields decrease to values optimal for excitation of electronic degrees of freedom and dissociation. ${ }^{3}$ As gas density increases, the discharge loses its uniformity and must be considered as a set of separate streamers.

The nanosecond surface dielectric barrier discharge was suggested for PAI/PAC due to the fact that even at relatively low values of energy deposition the decrease in the ignition delay time is significant, and so, efficient multi-point ignition can be achieved even at high gas densities. In nanosecond surface DBD, for a few $\mathrm{cm}$ of the high voltage electrode length, the deposited energy is dispersed over the set of 100-200 streamers. ${ }^{3}$ Another important advantages of the nanosecond discharge is that a typical time of discharge gap closing and of production of active species does not exceed tens and hundreds of nanoseconds, and that main processes of recombination and energy relaxation from electronically excited states are shorter than a typical time necessary for initiation of combustion.

Despite the fact that the physics of DBDs has being already developed during the last few decades there is a lot of unresolved questions. In particular, it is a question of energy distribution in space and time. The aim of the present work is (i) to study, in subnanoseconds and submillimeters scale, a distribution of energy in SDBD in air at atmospheric pressure; (ii) to demonstrate, on the example of n-heptane/oxygen mixture, that the nanosecond plasma can efficiently be used for modification of combustion kinetics in the region of cool flames.

\section{Experimental setup}

The experimental program was divided in two parts. The first part concerns the study of the nonequilibrium low temperature plasma of surface DBD in air at ambient pressure and temperature. The second part is devoted to plasma assisted ignition using nanosecond surface dielectric barrier discharge. The experiments on plasma assisted ignition were carried out at Rapid Compression Machine located in laboratory PC2A of University Lille1. The $\mathrm{n}^{-} \mathrm{C}_{7} \mathrm{H}_{16}: \mathrm{O}_{2}: \mathrm{N}_{2}=1.8: 19.6: 78.6$ mixtures were ignited with high pressure nanosecond DBD discharge.

\section{A. The discharge cells}

Dielectric barrier discharge (DBD) is a discharge originating in a discharge gap at the conditions when at least one of the electrodes is covered by a dielectric. The current in the DBD is limited by charging of the dielectric. One of the modifications of DBDs is a surface DBD (SDBD). In this case, the electrode system is a sandwich-like structure, consisting of the low-voltage electrode, covered with a thin, parts of mm, dielectric layer, and high-voltage (HV) electrode of a smaller surface, glued on the dielectric. The discharge starts from the $\mathrm{HV}$ electrode and propagates along the dielectric surface.

A few configurations of electrode systems for nanosecond SDBD initiation are considered in the paper. The classical airflow, pin-to-pin and interjacent configurations are used to study the discharge parameters and the morphology of SDBD. Coaxial SDBD is used to adapt the discharge to the geometry of a rapid compression machine (RCM) for plasma-assisted combustion experiments.

The electrode system was connected to the generator via a $30 \mathrm{~m}$ coaxial $50 \Omega$ cable. The high voltage (HV) pulse generator (FID Technology, FPG20-03NM) used in the experiments provided the following parameters: $2 \mathrm{~ns}$ front rise time, $20 \mathrm{~ns}$ pulse duration on the half-height and $\pm(12-30) \mathrm{kV}$ voltage range in the cable (see figure 2). All experiments related to the study of SDBD, as well as experiments on PAI in RCM were performed in a single shot regime without a gas flow.

Two calibrated custom made back current shunts (BCS) were soldered in the shield of the coaxial cable: one, BCS1, in the middle of the cable and another one, BCS2, $1 \mathrm{~m}$ apart from the HV generator. The details 


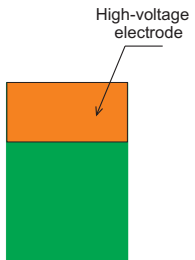

a)

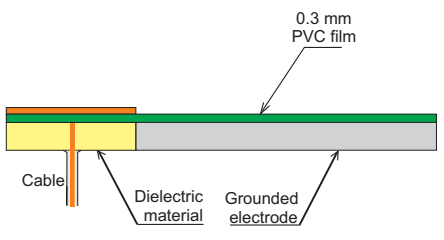

b)

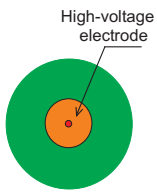

c)

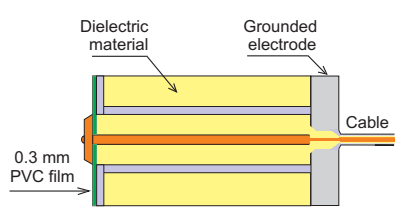

d)

Figure 1. Flat electrode system $(a, b)$ and cylindrical electrode system $(c, d)$.

of the technique can be found elsewhere. ${ }^{5}$ BCS1 was used to measure the voltage on the electrodes, the current through the electrodes and the deposited energy, and the BCS2 was used to synchronize the ICCD camera with the discharge. The signals from the BCSs were registered by a LeCroy WaveRunner $600 \mathrm{MHz}$ oscilloscope.

To study the spatial structure and the development of the surface discharge, the images of the discharge, integrated over the wavelength range $300-800 \mathrm{~nm}$, were recorded by PI-MAX4 Princeton Instruments ICCD camera (0.5 ns minimal gate) with Cosmicar Pentax YK5028 lens. A camera was triggered at different time delays from the beginning of the applied pulse to get the images of the discharge evolution.

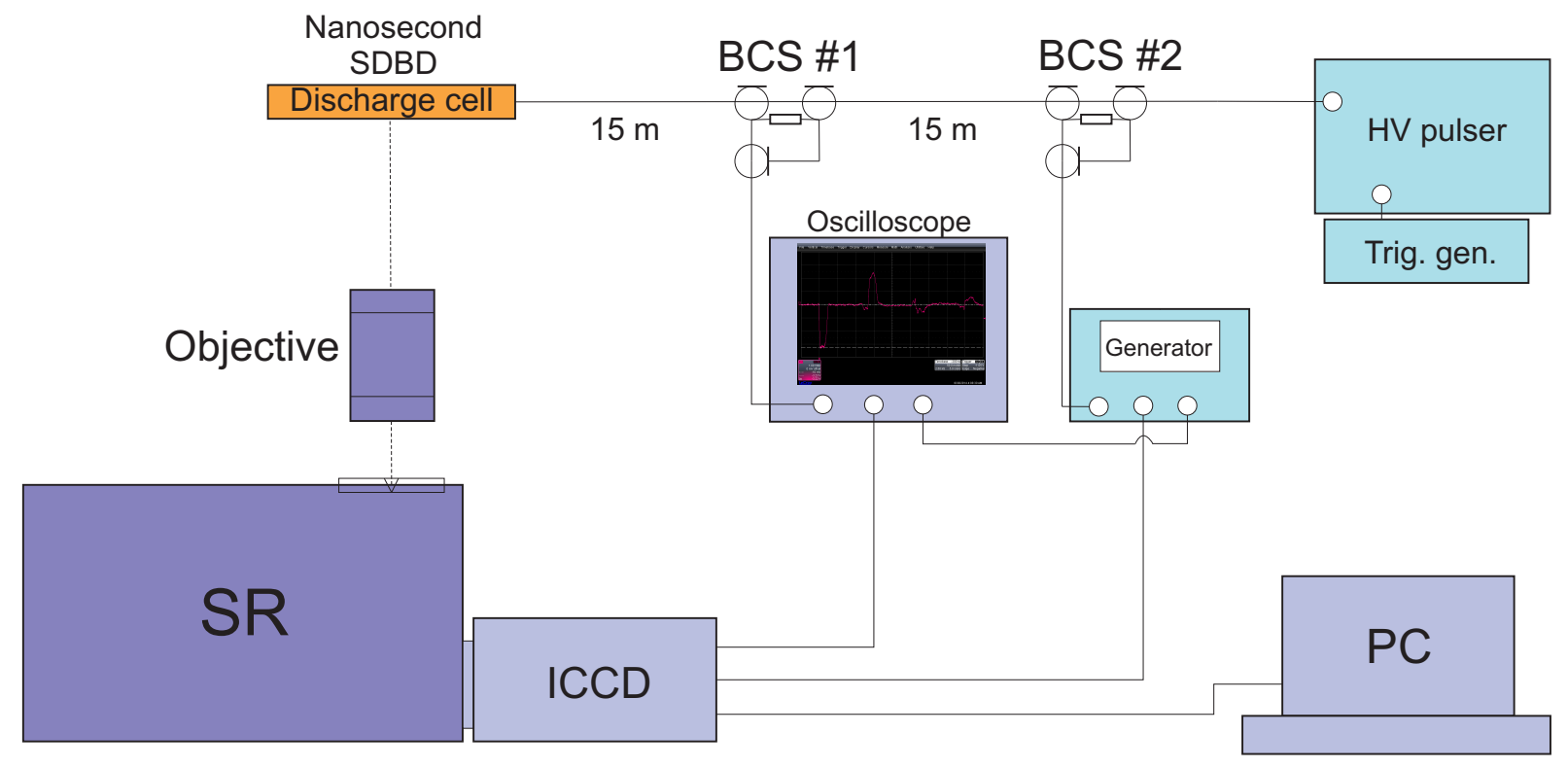

Figure 2. Scheme of experimental setup. BCS - back current shunt, SR - spectrometer, ICCD - ICCD camera, PC - computer.

In the case of a nanosecond SDBD, the main input to the emission in the considered spectral range is due to transitions of the second positive system of molecular nitrogen. For air, the quenching time of excited levels is determined by the collision of excited nitrogen molecules with molecular oxygen, the rate constant being equal ${ }^{6}$ to $k=2.7 \cdot 10^{-10} \mathrm{~cm}^{3} \mathrm{~s}^{-1}$. The efficient life time of $\mathrm{N}_{2}\left(\mathrm{C}^{3} \Pi_{u}\right)$ at the atmospheric pressure is $0.7 \mathrm{~ns}$, thus ICCD imaging adequately reflects the spatial structure of the discharge, and the resolution is limited by the camera gate.

The monochromator ACTON SP2500i Princeton Instruments combined with PI-MAX4 1024i ICCD camera was used to study the rotational structure of the second positive system of molecular nitrogen. Scheme of the experimental setup for study the optical emission from the discharge is represented schematically in figure 2. Resolution reached in the experiments with the grating $2400 \mathrm{l} / \mathrm{mm}$ and $50 \mu \mathrm{m}$ input slit width was $0.033 \mathrm{~nm}$. To select the emission from different regions of the discharge, the image of electrode system was projected on the entrance slit of monochromator by optical objective with achromatic UV lenses. The WinSpec software was used to select the sets of pixel lines that correspond to the region of interest. 
A similar discharge system but in cylindrical symmetry was used in experiments on plasma assisted ignition that were carried out in rapid compression machine (RCM) in PC2A laboratory of Lille University ${ }^{8-11}$ in $\mathrm{n}$-heptane containing mixtures.

\section{B. Rapid Compression Machine}

The Rapid Compression Machine of University of Lille 1 has been extensively described before ${ }^{10,12}$. It has a right-angle design, which ensures that the volume is kept strictly constant at the end of the compression at top dead center (TDC). The RCM combustion chamber is a cylinder, whose diameter is $50 \mathrm{~mm}$ and height is $17 \mathrm{~mm}$.

Temperatures around 600-650 $\mathrm{K}$ and gas pressures from 2 to 7 bar were obtained by compressing gas mixtures at given initial temperature and pressure. The coaxial SDBD electrode system was mounted in the end plate of the combustion chamber. A discharge was generated at a selected moment of time corresponding to the TDC point (top dead center, or point of maximal compression). The central coaxial high voltage (HV) electrode was ended by a bevel-edged brass disk, $2 \mathrm{~mm}$ in thickness and $20 \mathrm{~mm}$ in diameter. The low voltage electrode was made of aluminum. The inner diameter of the low voltage electrode was equal to the outer diameter of the $\mathrm{HV}$ electrode, and the outer diameter of the low-voltage electrode was equal to $46 \mathrm{~mm}$. A dielectric layer of PVC $0.3 \mathrm{~mm}$ in thickness was located between the electrodes. The angle between the high voltage electrode edge and horizontal plane was equal to $45^{\circ}$. The optical side window, $15 \mathrm{~mm}$ in diameter, was used to monitor optical emission in the visible range of spectra. The position of the electrode and RCM piston is given in figure 3 .

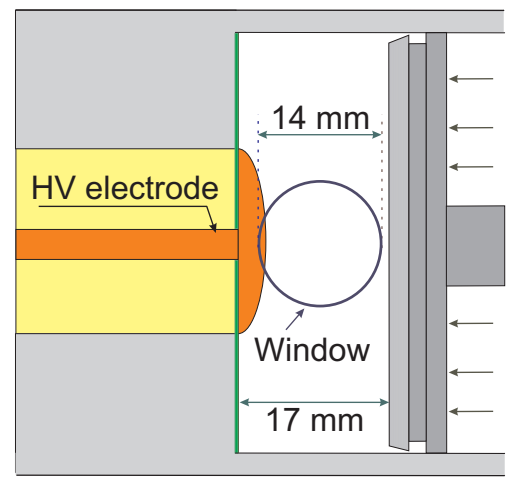

Figure 3. Optical access to the combustion chamber.

The amplitude and the shape of the incident high-voltage pulses were measured by a calibrated BCS1 installed, as mentioned above, into the cable shield break in the middle of the cable, $15 \mathrm{~m}$ apart from the combustion chamber. In the long cable, the pulse coming from the high-voltage generator and reflecting from the electrode system are separated in time. The energy deposition in the discharge can be calculated, with the accuracy of few percent, as a difference of energies of incident and reflected pulses. An addition series of the experiments have been performed to extract the losses in the cable and energy spent to the charging of the electrode system itself.

\section{Experimental results}

\section{A. Spatial and time energy distribution of the discharge}

Morphology of the discharge, distribution of specific deposited energy in time and in space, and ratio between gas heating and production of active species are key issues for plasma assisted ignition and combustion. At high pressures, specific deposited energy is directly related to the discharge morphology, the last one being a function of gas mixture composition and geometry of the system.

Nanosecond surface dielectric barrier discharge (nSDBD) in atmospheric pressure ambient air has been studied in the first part of this work. To study a possibility to optimize the energy release and the discharge configuration, adapting the last one to a particular combustion system, three different electrode configuration have been tested: classical airflow configuration, pin-to-pin surface configuration and interjacent one. All 
three systems are schematically represented in the upper line of figure $4 \mathrm{a}, \mathrm{c}$ and b respectively. The SDBD was initiated by $20 \mathrm{~ns}$ positive polarity high voltage pulses with a $30 \mathrm{kV}$ amplitude on the electrode. The experiments were made in a single-shot regime.

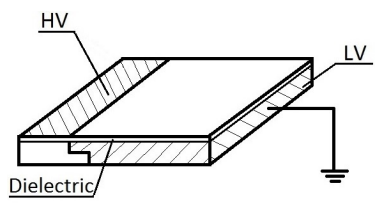

a
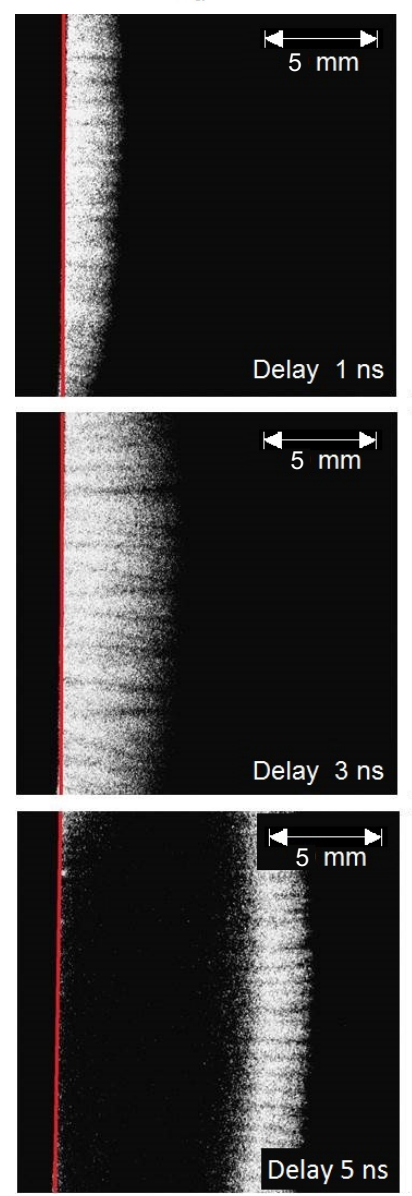

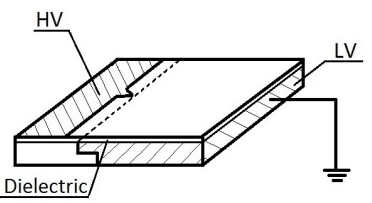

b
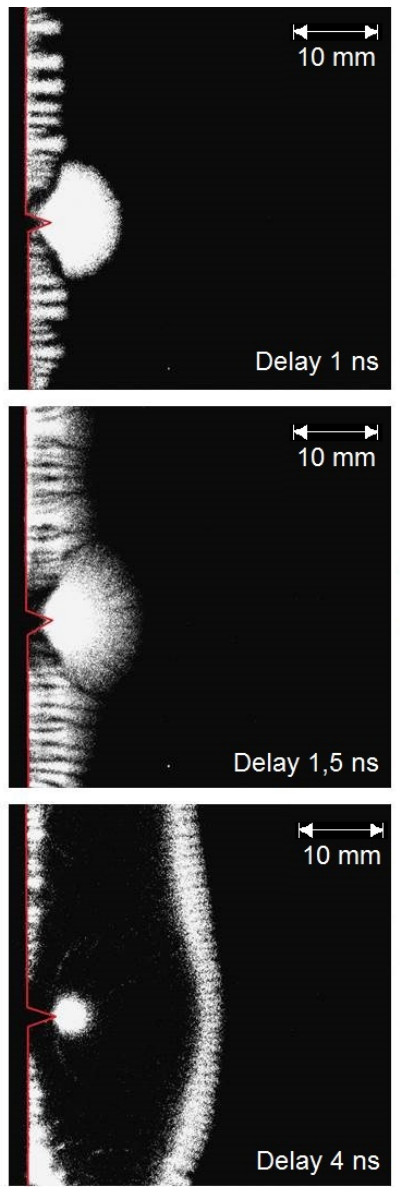

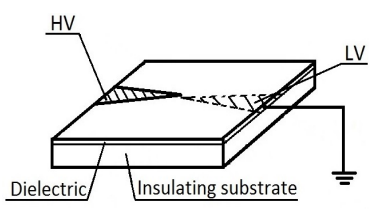

$\mathrm{C}$
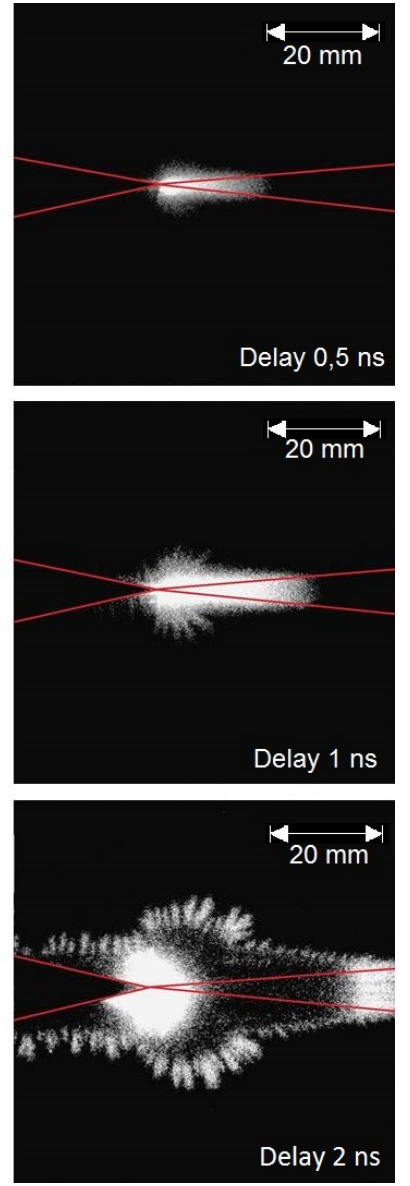

Figure 4. Evolution of SDBD in air for different geometries of the high voltage electrode in the system of flat electrodes. For all experiments the voltage of applied pulses was $30 \mathrm{kV}$ on the electrode. ICCD camera gate is $1 \mathrm{~ns}$.

The ICCD images taken with a camera gate of $1 \mathrm{~ns}$ and corresponding to the emission of the second positive system of molecular nitrogen are given by figure 4 . Time delay relative to the start of the emission in the system is given for each frame. Under conditions of the experiments, more then $90 \%$ of optical emission corresponds to the emission of the second positive system of molecular nitrogen $\left(\mathrm{N}_{2}\left(\mathrm{C}^{3} \Pi_{u}\right) \rightarrow \mathrm{N}_{2}\left(\mathrm{~B}^{3} \Pi_{g}\right)\right)$ with a threshold of excitation $\varepsilon=11.03 \mathrm{eV}$. Although the $\mathrm{N}_{2}$ emission does not reflect unambiguously the energy distribution in the system, it gives the idea about the distribution, in time and space, of the electrons with energy enough for direct excitation of $\mathrm{N}_{2}\left(\mathrm{C}^{3} \Pi_{u}\right)$-state.

In the classical airflow geometry (figure 4 a) with a metal strip as the high-voltage electrode, the SDBD starts as a set of synchronous streamers propagating along the dielectric in the direction perpendicular to the $\mathrm{HV}$ electrode, the velocity of propagation is being $(3.0 \pm 0.5) \mathrm{mm} / \mathrm{ns}$. The picture changes for the $\mathrm{HV}$ electrode with a sharp tip. The SDBD develops as a combination of "classical" set of parallel streamers with the same velocity and the discharge initiated at the point of the high electric field, at the vicinity of the electrode tip (figure $4 \mathrm{~b}$ ). The discharge starts from the pin with a velocity similar to the velocity of the 

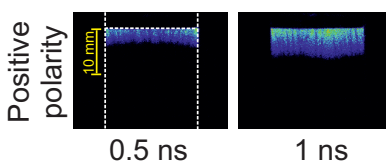

$1 \mathrm{~ns}$

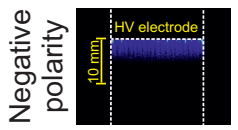

$0.5 \mathrm{~ns}$

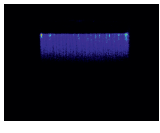

$1 \mathrm{~ns}$

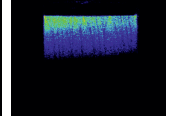

$1.5 \mathrm{~ns}$

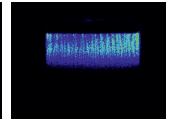

2 ns

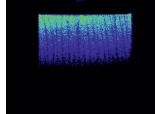

$2 \mathrm{~ns}$

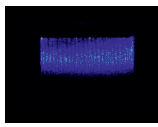

3 ns

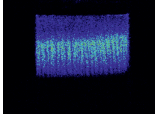

3 ns

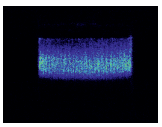

4 ns

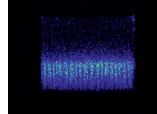

$4 \mathrm{~ns}$

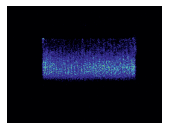

5 ns

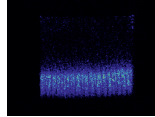

5 ns

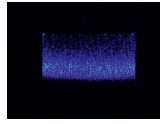

6 ns

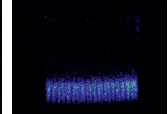

$6 \mathrm{~ns}$

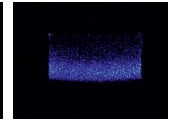

8 ns

Figure 5. ICCD imaging of classical air flow electrode system. Gate of the camera is $0.5 \mathrm{~ns}$.

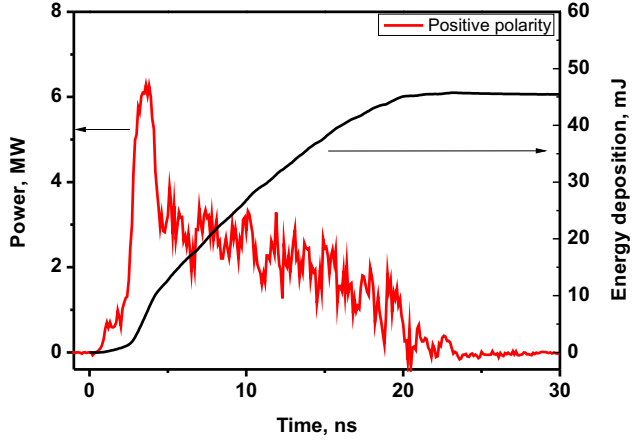

a)

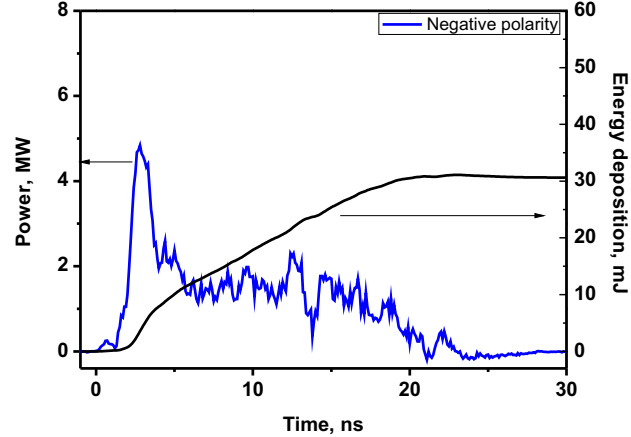

b)

Figure 6. Power and energy deposited into the discharges for (a) positive and (b) negative polarities.

discharge in pin-to-pin geometry, and then slows down to $(3.0 \pm 0.5) \mathrm{mm} / \mathrm{ns}$. In a few nanoseconds, both parts of the discharge merge and propagate as a single structure. This configuration can be interesting for the applications when it is necessary to start the chemical process in the local point(s) and to sustain it further along all the surface of the electrode. And finally, in the pin-to-pin configuration (figure $4 \mathrm{c}$ ) the discharge develops in the direction parallel to the axis of the pins, but relatively bright glow is observed around, consisting of the streamers propagating in perpendicular direction. This fact underlines a complex structure of the nanosecond SDBD, proving that pin-to-pin geometry does not represent a single streamer but another discharge with different characteristics. The velocity of the main part of the discharge propagating along the pin axis is equal to $(15 \pm 2) \mathrm{mm} / \mathrm{ns}$. So, modification of the electrode system can result in a significant re-distribution of specific deposited energy for different zones of the discharge. Combination of heat release and following hydrodynamic perturbations and of production of active species can be so optimally chosen to accelerate the ignition in the combustion chamber.

For more detailed study of the discharge, classical air flow configuration of SDBD was used. As it was mentioned above, the discharges propagates like a set of parallel streamers along the surface of dielectric layer. The dimensions of the streamers are different in the case of positive and negative polarity. For positive polarity, the optical diameter of the streamers changes between $0.7-1.2 \mathrm{~mm}$, whereas for negative polarity it is in the range $0.3-0.8 \mathrm{~mm}$. The velocities differ as well. For positive polarity, the discharge front starts from the high-voltage electrode with a velocity of $2.7 \mathrm{~mm} / \mathrm{ns}$, and slows down to about $0.7 \mathrm{~mm} / \mathrm{ns}$ in approximately $10 \mathrm{~ns}$. For negative polarity, discharge starts with a velocity equal to the velocity of a positive discharge; slows down to $1 \mathrm{~mm} / \mathrm{ns}$ in $3-4 \mathrm{~ns}$, and slows down further to about $0.15 \mathrm{~mm} / \mathrm{ns}$ at about $10 \mathrm{~ns}$ from the start of the discharge.

The total power deposited into the discharge is shown in figure 6 for both polarities. During the discharge, $45 \mathrm{~mJ}$ is deposited into gas for positive and $30 \mathrm{~mJ}$ - for negative polarity. The most considerable power dissipates in the gas during first $5 \mathrm{~ns}$ of the pulse, corresponding to a fast propagation of the streamers along the dielectric and charging of a "plasma" capacitance. After $\approx 7 \mathrm{~ns}$, the streamers slow down, and the energy provided by high-voltage electrode, decreases. 


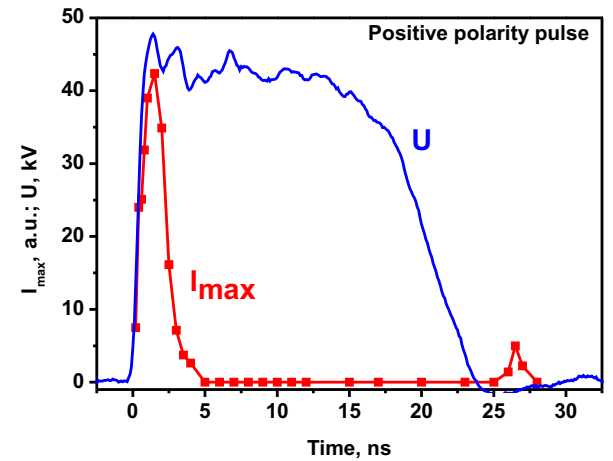

a)

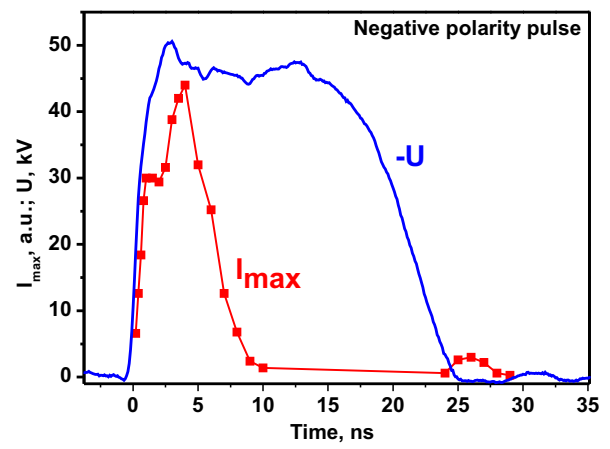

b)

Figure 7. The peak intensities of the (0-0) line of $2+$ system of molecular $\mathbf{N}_{2}$ for (a) positive and (b) negative polarities. Zone 0-1 $\mathrm{mm}$ form the high-voltage electrode.

Time- and space-resolved emission spectra of the second positive system of molecular nitrogen were taken near the high-voltage electrode and $5 \mathrm{~mm}$ apart. To identify the periods of time that can be used for analysis of spectrum, the peak intensities of the (0-0) spectral lines of the second positive system of molecular nitrogen near the high-voltage electrode were measured at different time instants; they are represented in figure 7 together with the voltage pulses. The difference between the positive and the negative polarities is clearly seen and correspond to ICCD images represented in Figure 5: (i) for both polarities, two peak of emissions are observed, corresponding to the rising front, during first $5 \mathrm{~ns}$ and to the falling edge of a pulse, at $t=25-20 \mathrm{~ns}$; (ii) for positive polarity, no emission is observed during the plateau of the pulse, while for negative polarity, the high intensity of emission is observed after the front, at $t=5-10 \mathrm{~ns}$. It should be noted that the synchronization of voltage and emission signals in figure 7 is artificial and corresponds to rising fronts of voltage and emission pulses.

\section{B. Population of the energy levels and rotational temperature distribution}

The main principle of the spark discharge is a local gas heating. In combustible mixtures the flame/exposion occurs with the following propagation of the combustion wave from the point of ignition. In contrast, in the
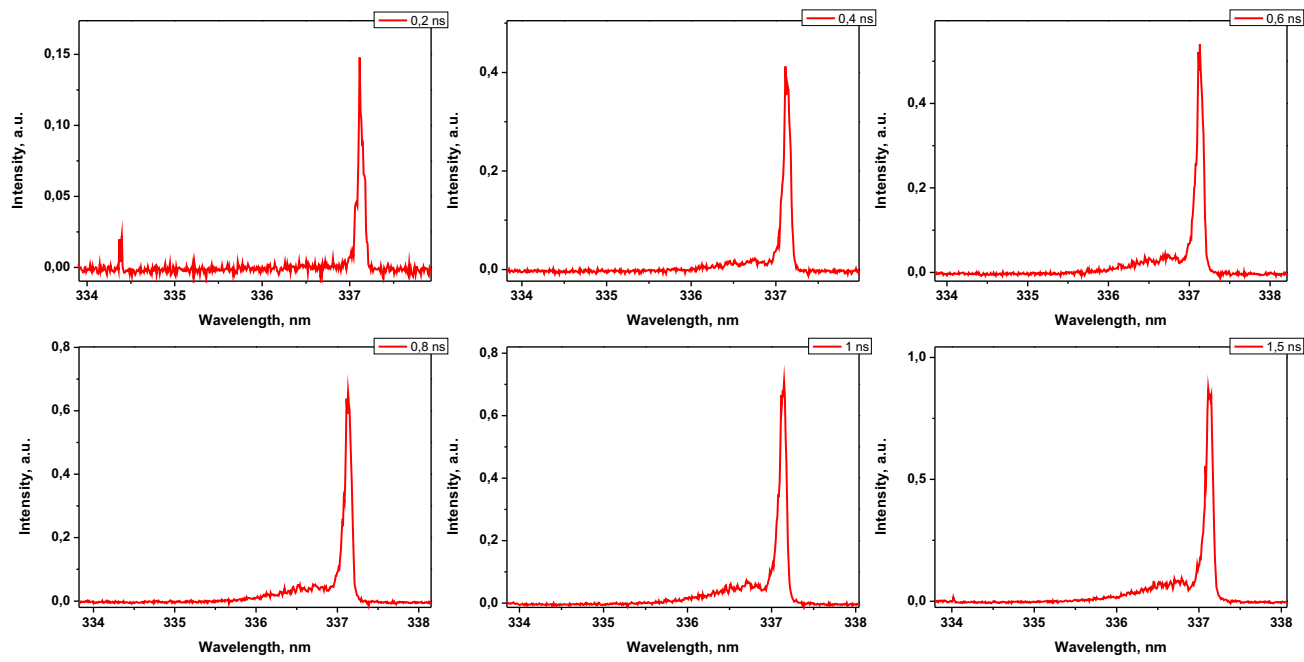

Figure 8 . The spectra of line $337,1 \mathrm{~nm}$ on the leading edge. Positive polarity of high-voltage pulses. 

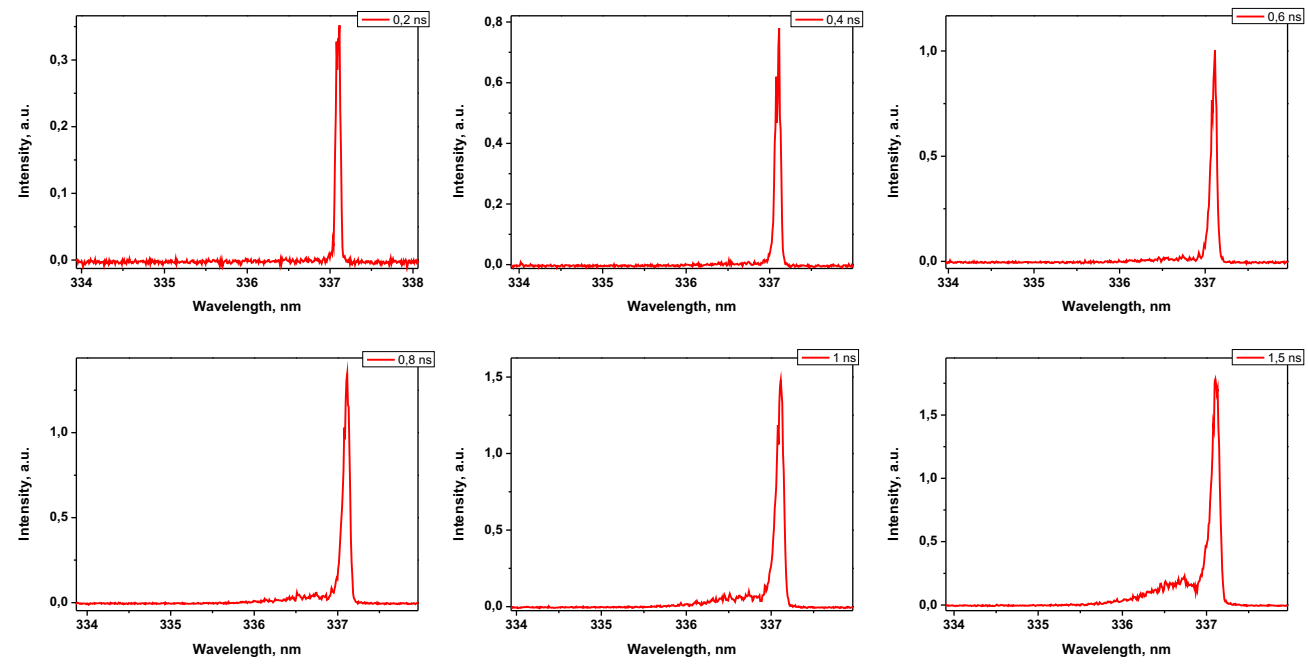

Figure 9. The spectra of line $337,1 \mathrm{~nm}$ on the leading edge. Negative polarity of high-voltage pulses.

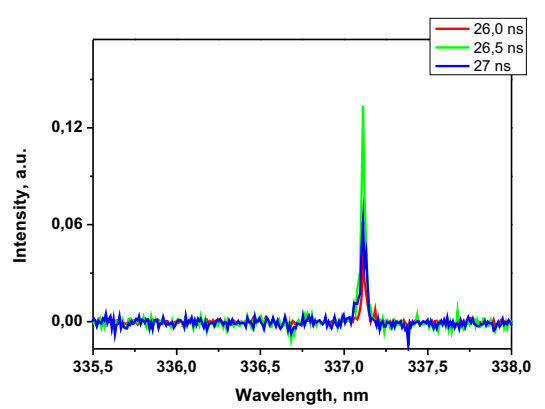

a)

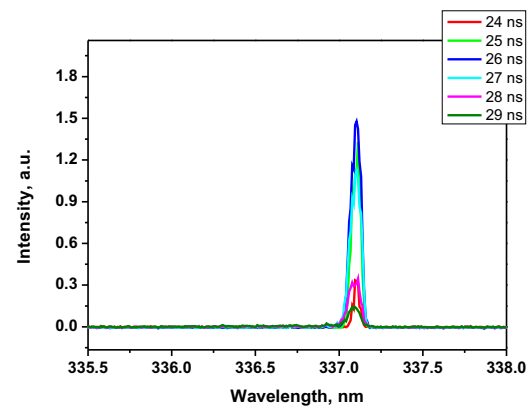

b)

Figure 10. The spectra of line $337,1 \mathrm{~nm}$ on the trailing edge of (a) positive and (b) negative polarities of applied high-voltage pulses.

case of non-equilibrium plasma the gas is not initially heated by discharge, e.g. electron energy goes mainly to excitation of the internal degrees of freedom that subsequently leads to the increase of the internal energy of the species. Use of internal energy for the initiation of a chemical transformation can be a more efficient method than that involving transformation initiated by thermal energy only. For more detailed analysis of the kinetics of plasma assisted combustion with non-equilibrium plasma it is necessary to know which part of the energy transforms in heat release and how this heat is distributed over the discharge or gas volume. For this purpose it would be a good start of this analysis to determine the rotational temperature distribution.

It was decided to measure the rotational temperature in different regions of electrode and in different moments of time. For this purpose the emission spectra of $2+(0-0)$ line were obtained with camera gate of $0.5 \mathrm{~ns}$; the spectra were taken each $0.1 \mathrm{~ns}$ with changeable delay. During the analysis of the spectra the significant non-equilibrium of the rotational population has been detected at the very beginning of the discharge initiation i.e. on the leading edge of the high-voltage pulse, and at the end of the pulse. Figures 8 and 9 demonstrate the rotational spectra of (0-0) lines of $2+$ system of molecular nitrogen on the leading edge of the pulse for positive and negative polarity respectively. The optical signal was obtained over the region 0-1 mm near high-voltage electrode. As one can notice, the $\mathrm{R}$-branch of rotational spectrum is mostly suppressed whereas the $\mathrm{P}$-branch is clearly seen. In a short time $(0.5-0.8 \mathrm{~ns}$ for the positive polarity and $0.8-1$ ns for the negative polarity) the spectra transform to a "common" shape corresponding to Boltzmann distribution over the rotational lines. 


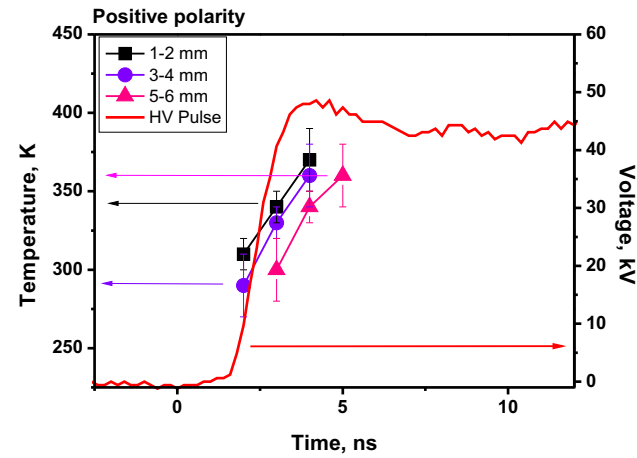

a)

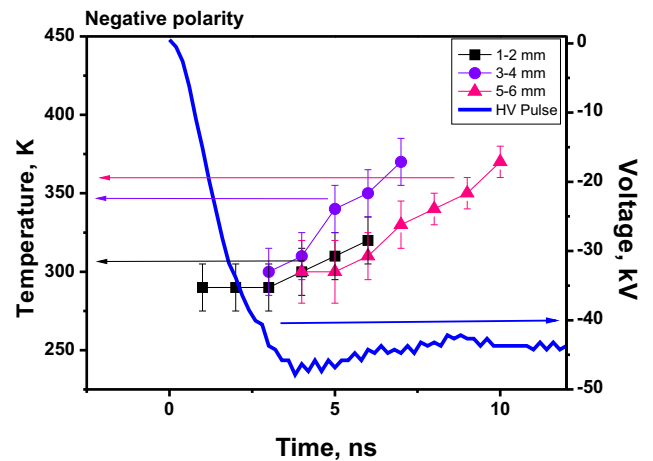

b)

Figure 11. Time resolved rotational temperature for (a) positive and (b) negative polarity of applied pulses, taken at different distances from the high-voltage electrode.

The non-equilibrium shape of the emission spectra can be caused by two reasons: (i) at the leading edge of the pulse, the very strong values of electric field in short period can split, due to Stark effect, observed rotational lines. In the front, the voltage changes by $12.5 \mathrm{kV}$ during $0.5 \mathrm{~ns}$, corresponding to ICCD camera gate. As the density of lines is very high near $337 \mathrm{~nm}$, a broad feature is appearing at the position but for other wavelength this will appear as a continuum decreasing at averaging the spectra; (ii) The mechanism of destruction of high rotational levels in electric field is related to the distortion of the potential energy of the nuclei in the field of the electrons, which leads to a decrease in the level of pre-dissociation by rotation and dissociation of molecules with high rotational levels due to the tunneling effect. A serious objection against the first mechanism is that the resolution of a used spectral system is relatively low, $0.033 \mathrm{~nm}$. The Stark splitting with following integration of the emission over the spectral resolution of the system, even for high electric fields, must not give such a serious difference for $\mathrm{P}$-branch and $\mathrm{R}$-branch of the spectra. The objection against the second mechanism is that the peculiarities in the spectra start with low $J$ numbers: the R-branch does not overlap with P-branch at $J>5$.

The same behavior of the spectral line has been observed on the trailing edge of the HV pulse. The emission of the discharge occurs only at near electrode region (not more faraway than $3 \mathrm{~mm}$ from HV electrode). The spectra represented in figure 10 were obtained over the region $0-1 \mathrm{~mm}$. The intensity of the emission for the negative polarity pulse is higher than for the positive polarity. The effect of R-branch suppression at the trailing edge of the pulse is observed for a longer period of time then on the leading edge.

The rotational temperature $T_{\text {rot }}$ was measured from the emission spectra of $(0-0)$ line of $2^{+}$system for the time interval where it was possible to superimpose a Boltzmann plot calculated by Specair code ${ }^{13}$ and experimentally obtained rotational spectra and to get a reasonable information for the R-branch of the spectra. To increase the signal-to-noise ratio, the spectra were obtained over the regions $0-2,2-4$ and 4-6 mm counting from the HV electrode, and the camera gate was set to $1 \mathrm{~ns}$. The temperature timedependent distribution and corresponding HV pulses are shown in figure 11. For both positive and negative polarity of the high voltage pulse the temperature increase does not exceed $100 \mathrm{~K}$ respectively to the initial temperature that was equal to $290 \mathrm{~K}$. It can be also noted that there is a difference between the temperature measured at different distances: in general, temperature decreases with distance from the electrode.

\section{Plasma assisted ignition of $\mathbf{n}$-heptane containing mixtures}

Providing moderate temperature increase and elevated densities of atoms and radicals, nanosecond multistreamer discharge can be used as initiator of combustion at high pressures. ${ }^{8}$ At high pressures, action of nanosecond DBD leads to multi-spot ignition in the vicinity of the high-voltage electrode with a following propagation of the combustion wave in the combustion chamber of a rapid compression machine. In the present work, a capability of a nanosecond discharge to produce a volumetric excitation and to trigger a cool flame was tested on example of a stoichiometric n-heptane/air mixture.

To get a volumetric initiation of combustion, relatively low pressures were used in the RCM. It was 


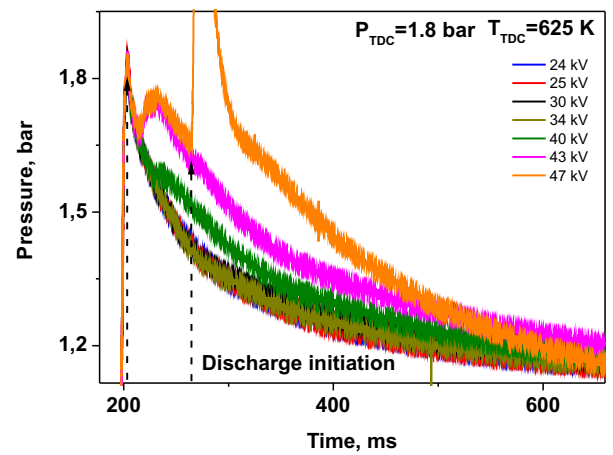

a)

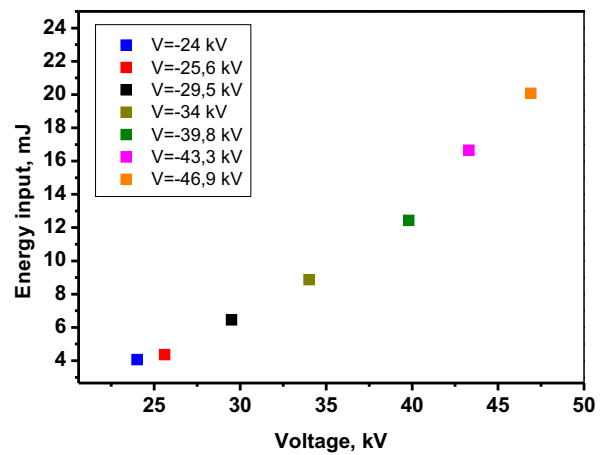

b)

Figure 12. (a) Modification of pressure traces under the action of nanosecond surface dielectric barrier discharge, $\mathbf{n}-\mathbf{C}_{7} \mathbf{H}_{16}: \mathrm{O}_{2}: \mathbf{N}_{2}=\mathbf{1 . 8 : 1 9 . 6 : 7 8 . 6}$ mixture, $P_{T D C}=1.8 \mathrm{bar}, T_{C}=626 \mathrm{~K}$. Two nanosecond pulses, at time instance $210 \mathrm{~ms}$ and $270 \mathrm{~ms}$, are applied to the system. Negative polarity pulses, voltage amplitude is between 24 and $46.9 \mathrm{kV}$. (b) deposited energy in the first pulse as a function of voltage amplitude on the high-voltage electrode in $\mathrm{n}$-heptane-containing mixture.

possible to get a discharge-triggered cool flame with further transition to a regular heptane-air flame by two different experimental ways: increasing deposited energy (when increasing applied voltage) or increasing initial pressure of the gas mixture. These two ways are demonstrated by figures 12-14.

Pressure profiles in RCM combustion chamber at initial pressure 1.8 bar and initial temperature $626 \mathrm{~K}$ are given by figure 12. In this particular regime, two successive discharge pulses were used, the first pulse at the point of maximum compression (corresponds to $t=200 \mathrm{~ms}$ in the figure), and the second pulse $60 \mathrm{~ms}$ later. Slight changes of gas pressure become visible at $19-34 \mathrm{kV}$ of high voltage amplitude on the electrode. At $40 \mathrm{kV}$, well pronounced pressure increase $25 \mathrm{~ms}$ after the point of maximum compression is clearly seen. At $43 \mathrm{kV}$ pressure increase starts earlier, $10 \mathrm{~ms}$ after the first discharge. Second discharge slightly increases

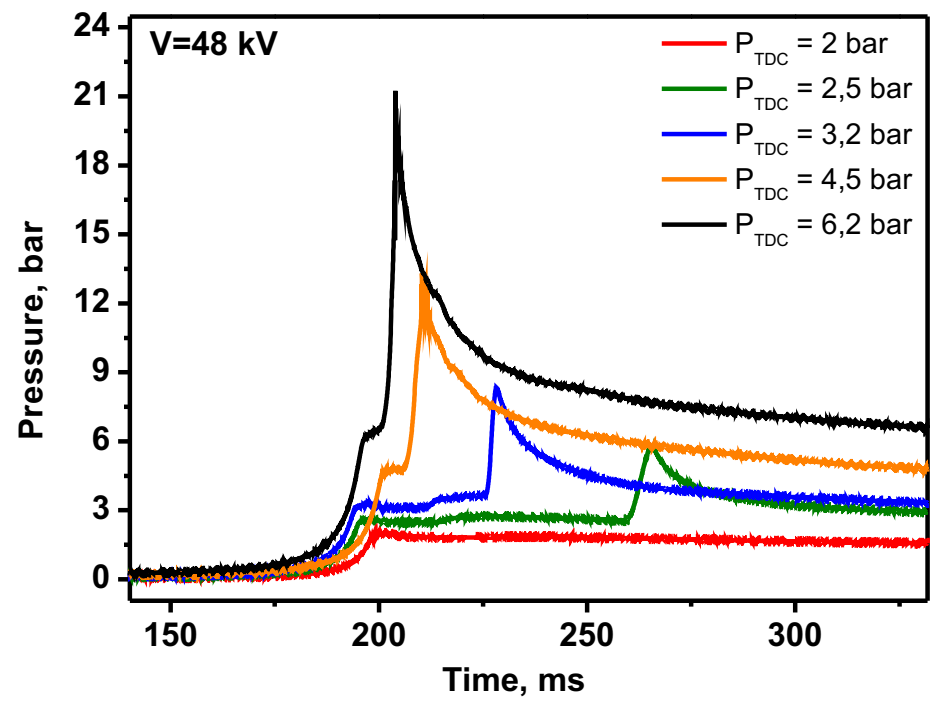

Figure 13. Modification of pressure traces under the action of nanosecond surface dielectric barrier discharge, $\mathrm{n}-\mathrm{C}_{7} \mathrm{H}_{16}: \mathrm{O}_{2}: \mathrm{N}_{2}=1.8$ :19.6:78.6 mixture, different initial pressures. Negative polarity pulses, voltage amplitude is $46.9 \mathrm{kV}$. 

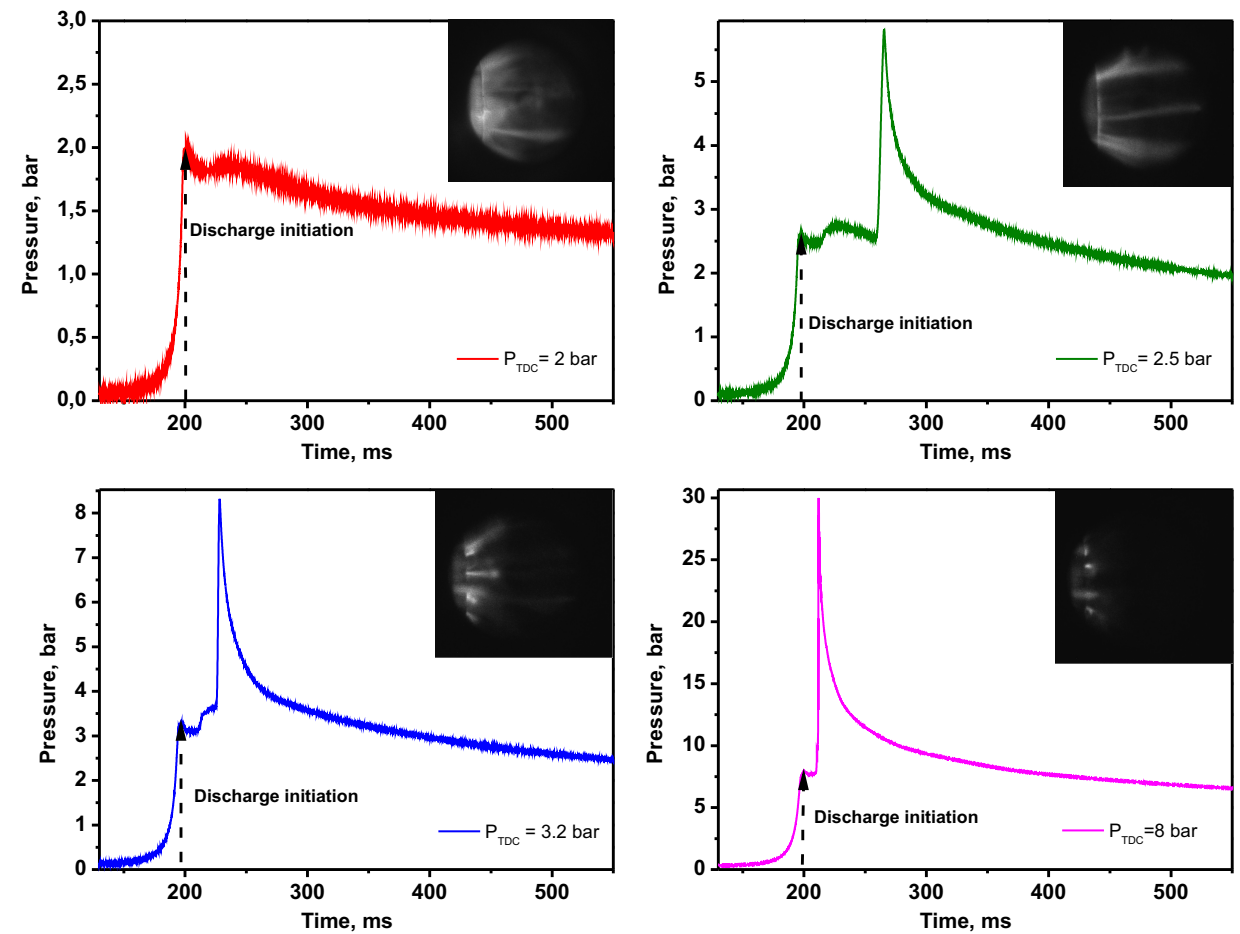

Figure 14. Pressure profiles in $\mathbf{n}-\mathrm{C}_{7} \mathrm{H}_{16}: \mathrm{O}_{2}: \mathbf{N}_{2}=\mathbf{1 . 8 : 1 9 . 6 : 7 8 . 6}$ mixture at $U=-46.9 \mathrm{kV}$ and corresponding ICCD images of the discharge. ICCD gate is $100 \mathrm{~ns}$.

the pressure. Finally, at $47 \mathrm{kV}$, the second pulse triggers a regular flame, providing significant, more than twice, pressure increase in the combustion chamber. Voltage increase from 24 to $47 \mathrm{kV}$ corresponds to deposited energy increase from 4 to $20 \mathrm{~mJ}$. At $P=1.8$ bar and $T=626 \mathrm{~K}$, two-phase ignition was possible only when triggered by a sequence of pulses.

Single pulse experiments at different pressures and at maximum voltage, $U=48 \mathrm{kV}$, have been carried out. When pressure rises from 2.0 to 6.2 bar, the pressure trace modifies from a trace corresponding to a cool flame triggered by the discharge (at 1.8 bar) to a trace corresponding to ignition near the electrode and following combustion wave propagation, similar to regimes observed in methane- and n-butane-containing mixtures. ${ }^{10}$ The sequence of pressure profiles is given by figure 13, and pressure profiles with corresponding ICCD images taken during the discharge are presented in figure 14. The camera gate was $100 \mathrm{~ns}$ so that all the light emitted during the discharge pulse was recorded. The wavelength range was determined by combination of transmission of the optical window and of the broad band filter, and was equal to $300-400 \mathrm{~nm}$. It was found that at high pressure ( 8 bar) the discharge develops mainly in the plane of the electrode system. At low pressure (2 bar) the discharge is volumetric: bright longitudinal structures developing from the electrode situated at the left hand side of the frame are clearly seen at $P=2$ bar. For intermediate pressures (2.55 bar) the discharge is still volumetric; two-phase ignition, with delay time changing as a function of pressure, is observed in the system.

\section{Conclusions}

Nanosecond pulsed discharge with DBD configuration of electrodes was studied in air at ambient pressure and temperature and used in experiments in rapid compression machine to ignite n-heptane:air mixture.

It was shown that discharge morphology can be optimized by changing the configuration of the electrode system: size of streamers and direction of the discharge propagation, energy release and velocity of the discharge front depends significantly upon the electrodes geometry.

One of the most important physical value characterizing the discharge is energy deposition. The power 
and the energy deposition were measured for classical airflow configuration; it was shown that the main energy release takes place at first 5-7 ns, during discharge propagation along the dielectric. Significant difference was observed between positive and negative polarity pulses: for the same voltage amplitude and pulse shape, the deposited energy is 1.5 higher for a positive polarity discharge.

Time- and space-resolved emission spectra were taken for the nSDBD discharge in air. It was shown that rotational spectra of $\mathrm{N}_{2}\left(\mathrm{C}^{3} \Pi_{u}\right) \rightarrow \mathrm{N}_{2}\left(\mathrm{~B}^{3} \Pi_{g}\right)$ transition are strongly non-equilibrium an the rising front and trailing edge of the high voltage pulse. Rotational temperature can be subtracted from the spectra a few nanoseconds after the rising front; it was shown that for both positive and negative polarity discharge temperature increase on the interval 0-10 ns does not exceed $100 \mathrm{~K}$, the temperature increase exhibits maximum near the high-voltage electrode.

Significant difference is observed in the behavior of the discharge when a small, a few percent, density of hydrocarbons is added to the mixture, at moderate (about $625 \mathrm{~K}$ ) heating. Discharge images in the combustion chamber of a rapid compression machine show that the discharge has a volumetric character under conditions when the discharge in air at the same gas density develops near the electrode surface.

Two-stage ignition, exhibiting a well-pronounced cool flame and a "regular" (or main) flame has been observed in $\mathrm{n}-\mathrm{C}_{7} \mathrm{H}_{16}: \mathrm{O}_{2}: \mathrm{N}_{2}=1.8: 19.6: 78.6$. The pressure traces were changed with initial pressure at constant high voltage amplitude or with voltage at constant initial gas pressure. Obtained experimental conditions can be further used to study cool flame chemistry initiated by non-equilibrium plasma of a nanosecond discharge.

\section{Acknowledgements}

The work was partially supported by French National Agency, ANR (PLASMAFLAME Project, 2011 BS09 025 01), AOARD AFOSR, FA2386-13-1-4064 grant, and by Plas@Par Projects. The author are thankful to N. Sadeghi and to B.P. Lavrov for helpful discussions.

\section{References}

\footnotetext{
${ }^{1}$ Vasilyak L M, Kostyuchenko S V, Kudryavtsev N N and Filyugin I V 1994 Fast ionization waves under electrical breakdown conditions Physics-Uspekhi 37(3) 247-268

${ }^{2}$ Tardiveau P, Moreau N, Bentaleb S, Postel C and Pasquiers S 2009 Diffuse mode and diffuse-to-filamentary transition in a high pressure nanosecond scale corona discharge under high voltage J. Phys. D: Appl. Phys. 42175202

${ }^{3}$ Starikovskaia S M, Anikin N B, Pancheshnyi S V, Zatsepin D V and Starikovskii A Yu 2001 Pulsed breakdown at high overvoltage: development, propagation and energy branching Plasma Sources Sci. Technol. $10344-355$

${ }^{4}$ Kosarev I, Khorunzhenko V, Mintoussov E, Sagulenko P, Popov N and Starikovskaia S, 2012 A nanosecond surface dielectric barrier discharge at elevated pressures: time-resolved electric field and efficiency of initiation of combustion. Plasma Sources Sci. Technol. 20045002

${ }^{5}$ Anikin N B, Starikovskaia S M, Starikovskii A Yu 2004 Study of the oxidation of alkanes in their mixtures with oxygen and air under the action of a pulsed volume nanosecond discharge Plasma Physics Reports 30/12 1028 - 1042

${ }^{6}$ Pancheshnyi S V, Starikovskaia S M and Starikovskii A Yu 1998 Measurements of rate constants of the $\mathrm{N}_{2}\left(\mathrm{C}^{3} \Pi_{u}, v^{\prime}=0\right)$, and $\mathrm{N}_{2}^{+}\left(\mathrm{B}^{2} \Sigma_{u}^{+}, v^{\prime}=0\right)$ deactivation by $\mathrm{N}_{2}, \mathrm{O}_{2}, \mathrm{H}_{2}, \mathrm{CO}$ and $\mathrm{H}_{2} \mathrm{O}$ molecules in afterglow of the nanosecond discharge Chemical Physics Letters 29 523-527

${ }^{7}$ Boumehdi M A, Stepanyan S A, Shcherbanev S A, Starikovskaia S M, Desgroux P and Vanhove G 2014 Ignition of combustible mixtures by a surface nanosecond discharge in a rapid compression machine 35th International Symposium on Combustion, work-in-progress paper 3-8 August 2014, San Francisco, California, USA.

${ }^{8}$ Stepanyan S A, Boumehdi M A, Vanhove G, Desgroux P and Starikovskaia S M 2013 Time-resolved electric field measurements in nanosecond surface dielectric discharge. Comparison of different polarities. Ignition of combustible mixtures by surface discharge in rapid compression machine Proc. of 51st AIAA Aerospace Sciences Meeting 7-10 January 2013, Grapevine, Texas, AIAA-2013-1053

${ }^{9}$ Boumehdi M A, Stepanyan S, Starikovskaia S, Desgroux P and Vanhove G 2013 Plasma assisted ignition inside a rapid compression machine Proc. of European Combustion Meeting, 25-28 June 2013, Lund, Sweden

${ }^{10}$ Boumehdi M A, Stepanyan S, Desgroux P, Vanhove G and Starikovskaia S 2014 Ignition of methane- and n-butanecontaining mixtures at high pressures by pulsed nanosecond discharge. Accepted for publication

${ }^{11}$ Boumehdi M A, Stepanyan S A, Shcherbanev S A, Starikovskaia S M, Desgroux P and Vanhove G 2014 Ignition of combustible mixtures by a surface nanosecond discharge in a rapid compression machine 35th International Symposium on Combustion, work-in-progress paper 3-8 August 2014, San Francisco, California, USA.

${ }^{12}$ Vanhove G, Petit G, Minetti R 2006 Combust. Flame 145 521-532

${ }^{13}$ Laux C O 2002 Radiation and nonequilibrium collisional-radiative models. Physico-Chemical Modeling of High Enthalpy and Plasma Flows (von Karman Institute Lecture Series 2002-07) ed. D Fletcher et al (Rhode-Saint-Genese, Belgium: Von Karman Institute)
} 